\section{So near, but yet so far}

\author{
Human Evolution Through \\ Developmental Change \\ by Nancy Minugh-Purvis \& \\ Kenneth J. McNamara \\ Johns Hopkins University Press: 2002. 536 pp.

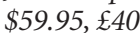

\section{Bernard Wood}

"Are humans really our closest ancestors?" If there were a newspaper for chimpanzees, this might well be one of its headlines. But there are no chimp news barons because the phenotypes and behaviours of chimps and modern humans are critically different. Yet many lines of evidence suggest that chimps and humans evolved from a common ancestor that lived between 5 million and over 10 million years ago. The challenge faced by those engaged in human evolution research is to reconstruct that common ancestor and then flesh out the details of its transformation into modern humans.

The common ancestor of chimps and humans was, of course, neither a chimp nor a modern human. But there are good reasons to think that many aspects of living chimps, including the timing and the sequence of events in their growth and development, such as weaning infants or changes in dentition, are reasonable proxies for the primitive condition in the common ancestor of chimps and humans.

Evolutionary change is brought about by altering growth and development. These changes can be restricted to a single structure, such as the hand, or they can be more or less global in their scope. These more general changes to the relative timing and the rate of development are subsumed into the term 'heterochrony'. Speeding up or slowing down the growth of the whole, or a substantial part, of an animal can change both the size and the shape of the evolved descendant.

Size, shape and timing are relatively crude ways of comparing ancestors and descendants, but they have the advantage of being relatively easy to measure, and we can translate them into changes in the onset, duration and offset time of cellular activity. Until molecular developmental biologists can point to the specificgenetic mechanisms that determine the way in which an individual's genome is translated into its phenotype, we may have to be satisfied with comparing the results of cellular activity, such as dental microstructure.

In this volume the editors address three main topics. The first is a broad-brush review of the links between development and evolution. The second is advertised as the evolution of life history within the higher primates, but in effect it concentrates on the developmental changes that underline phenotypic differences between living primates, with only a few intrepid authors tackling the thorny problem of reconstructing the evolutionary history of these differences. The third and final section concentrates on a few specific examples of how understanding the developmental sequence can inform hypotheses about the developmental basis of morphological differences between fossil hominin species.
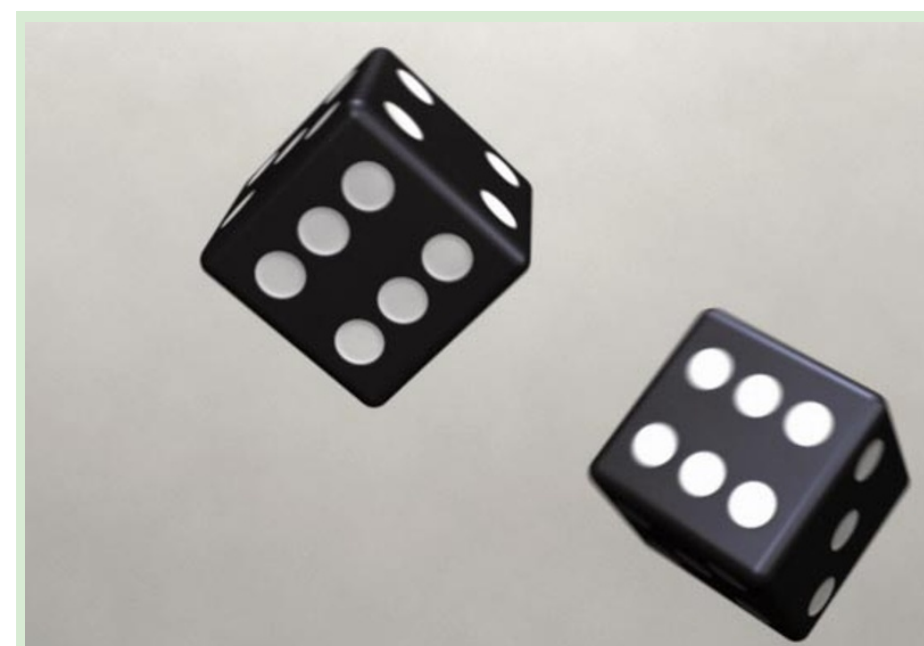

\title{
The dance of chance
}

Does God play dice? Is reality slave to chance? British artist Tim O'Riley responded to his confrontation with quantum theory during a recent visit to CERN, the European laboratory for particle physics, with a video installation of two dice orbiting each other in an endless loop, postponing indefinitely the chance moment of their fall. The video is part of the "Signatures of the Invisible" exhibition at the Gulbenkian Museum in Lisbon, which runs until 19 January.
Several themes emerge from the diverse contributions. The first is the confirmation that modern humans did not evolve from a more chimp-like precursor, because of some relatively simple global retention of juvenile characteristics. Distinctive sets of transformations seem to underlie each major change in morphology or life history. The second theme is the emphasis given in the volume to the evolution of the distinctive life history of modern humans. The pace and tempo of development is very different in modern humans and chimps. Like most primates, chimps have a general retardation of development (an extended infanthood), but modern humans have taken that trend to apparently unique levels of ontogenetic sloth. Even when the sequence of developmental events is the same, modern humans track through those stages much more slowly than chimps. Indeed, we have so effectively brought forward the time of weaning from the six or seven years we would predict from our body mass to about three years that we have had to invent a new term - childhood - to describe the stage in our life history between weaning and the end of brain growth and the eruption of the first molar teeth.

The third theme is the comprehension of just how difficult it will be to trace the evolutionary history of our unique phenotype and life history. Several studies of fossil evidence demonstrate that the phenotypic differences between modern humans and Neanderthals are established early in development. But it will be a long time before we have comparably sized samples for the taxa that are found earlier in the human fossil record. Only then will we have enough evidence to afford researchers equivalent opportunities to trace the ontogeny of what we hypothesize are taxonomically significant morphological differences between early hominin species.

Working out when, and in what context, the various distinctive aspects of modern human morphology and life history evolved is a formidable challenge. But unless that challenge is accepted and faced, we will remain ignorant about the evolutionary history and adaptive context of many of the most important components of our humanity.

Bernard Wood is in the Department of Anthropology, George Washington University, Washington DC 20052, USA.

More on human evolution

The Speciation of Modern Homo sapiens

edited by T. J. Crow

Oxford University Press, $£ 29.50$

The Neanderthal's Necklace: In Search of the First Thinkers

by Juan Luis Arsuaga

Four Walls Eight Windows, \$25.95 